\title{
THE CONVERSE OF WARING'S PROBLEM
}

\author{
BY L. E. DICKSON
}

1. Introduction. In the most general Waring problem we are given a set of integers $\geqq 0$ and seek $k$ such that every integer (or every sufficiently large integer) is a sum of $k$ numbers of the set. We then call the set a $k$-set. In the converse problem, $k$ is given and we seek all $k$-sets. There exist infinitely many $k$-sets; for example, (1) and (2).

In case every integer $\geqq 0$ is a sum of $k$ numbers of a set, we call the latter a universal $k$-set. It must contain 0 and 1 . By way of introduction, we construct some universal 2 -sets.

I. As the $n$th element of the set choose the least integer which is not a sum of any two of the first $n-1$ elements. The set is composed of 0 and all positive odd integers.

II. The set with $0,1,2$ and later elements chosen as in $I$ is composed of $0,1,2+3 x,(x=0,1, \cdots)$. It is a universal 2 -set.

III. After 0 and 1 choose the $n$th element as in I when $n$ is odd, but subtract 1 from the least when $n$ is even. We get the universal 2-set composed of $0,1,3+6 x, 4+6 x,(x=0,1, \cdots)$.

IV. After 0 and 1 employ blocks of three elements. Those in a block are odd (least as in I), odd (least), even (least less 1 ). We get the universal 2 -set $0,1,3+10 x, 5+10 x, 6+10 x,(x=0$, $1, \cdots)$.

V. Our aim here is to construct a bizarre 2-set. After 0, 1 employ blocks of $2,3,4, \cdots$ elements, where the last element of a block is even and the others are all odd, while the $n$th element is either the least or 1 less than the least integer which is not a sum of any two of the first $n-1$ elements. We get the set $0,1,3,4,9,11,16,21,23,27,28,33,35,39,41,46$, $53,59,65,71,77,82,83,89,95,97,101,107,114, \quad 119,125$, $127,133,139,145,151,156,163,169,175,181,187,193,199$, $205,212, \quad 217,219,225,231,237,243,249,255,261,266, \quad 267$, 273, 279, 285, 291, 297, 303, 309, 311, 317, 322, 329, etc.

Unlike I-IV, there is apparently no simple independent definition of this 2 -set. If we take the first elements of the successive blocks and form their differences of the second order, 
we obtain a highly irregular sequence (including a negative number).

2. Theorems regarding 2-Sets. THEOREM 1 . If $m \geqq 2,0 \leqq k \leqq m$, the set

(1) $0, \cdots, k-1, k+1, \cdots, m-1, k+m x,(x=0,1, \cdots)$,

is a universal 2-set.

The sums by 2 include $j+m x,(j=k, \cdots, k+m-1)$, which give all integers $\geqq k$. For $m=2, k=1$, we have I. If $m=2, k=0$ or 2 , the set is 1 and all even integers $\geqq 0$.

If $k \leqq m-3$, we obtain a universal 2 -set if we replace element $m-1$ in (1) by $2 m-1$. If either $k \leqq m-2$ or $k \geqq 4$, we obtain a universal 2 -set if we replace element 2 in (1) by $m+2$.

Theorem 2. If $m \geqq j+1$, we have the universal 2 -set

$$
0, \cdots, m-1, m+j+m x, \quad(x=0,1, \cdots) .
$$

If $j>0, m \geqq 2 j+2$, the set (2) with element $j$ deleted is a universal 2-set.

3. Simplification of the Problem. The problem to find all $k$-sets which involve a single linear function $n+m x$ reduces essentially* to the determination of integers $A, B, \cdots$, such that those sums by $k$ of $n, A, B, \cdots$, which involve $n$, include a complete set of residues modulo $m$.

In case $n$ is prime to $m$, we can find integers $a, b, \cdots$, such that $A \equiv n a, B \equiv n b, \cdots,(\bmod m)$. Then those sums by $k$ of $1, a, b, \cdots$, which involve 1 , include a complete set of residues modulo $m$. Hence if $n$ is prime to $m$, the problem reduces to the case $n=1$.

4. Determination of the 3-Sets. We readily determine all 3-sets

$$
0, a, b, 1+m x, \quad(x=0,1, \cdots) .
$$

From the sums by three involving $m$ we subtract 1 and get

$$
0,1,2, a, b, a+1, b+1,2 a, a+b, 2 b \text {. }
$$

These ten numbers shall include a complete set of residues modulo $m$, whence $m \leqq 10$.

* Omitting the evaluation of the (small) limit beyond which every integer is a sum of $k$ elements of the set. 
First let $m$ be even. Then the parity of a residue is invariant. The set (4) is symmetric in $a$ and $b$. Hence the following two cases are exhaustive:

$$
\text { if } a \equiv b(\bmod 2) \text {, exactly three of }(4) \text { are odd; }
$$

(6) if $a$ is even, $b$ odd, the odd (4) are $1, b, a+1, a+b$.

In any complete set of residues modulo $m$ ( $m$ even), exactly half of the terms are odd. Hence $m \neq 10$.

Let $m=8$. It suffices to treat case (6). The four odd in (6) must be congruent modulo 8 to $1,3,5,7$ in some order. By their sums, $a+b \equiv 3(\bmod 4)$. If $b \equiv 3-a(\bmod 8)$, we see from (6) that $a+1$ and 3-a must be congruent to 5, 7 in some order, whence $a \equiv 4$ or 6 . Finally, if $b \equiv 7-a$, then $a+1,7-a$ must be congruent to 3,5 , whence $a \equiv 2$ or 4 .

TheOREM 3. If $m$ is even and $>8$, there is no 3-set of type (3). If $m=8$, (3) is a 3-set if and only if $a, b$ is one of the pairs 2, $5 ; 3,4 ; 4,7 ; 5,6$.

Let $m=9$. If $a \equiv 0$ or 1 , there are two pairs of duplicates in (4). If $a \equiv 2$, the sum of the numbers in (4) with the duplicate $a$ omitted is $13+5 b$. But the sum of any complete set of residues modulo 9 is $\equiv 0$. Hence $b \equiv 1$ is a second duplicate. Similarly, $a \neq \equiv$ or 8 . Hence $a$ and by symmetry also $b$ must be chosen from $3,4,6,7$.

Let $a=3$. Then (4) are congruent to $0-4,6, b, b+1, b+3,2 b$. If $b=5, b+1 \equiv 6,2 b \equiv 1$ give two pairs of duplicates. If $b=6$, then $2 b \equiv 3$. But $b=4$ and 7 yield 3 -sets.

Theorem 4. If $m=9$, (3) is a 3-set if and only if $a, b$ is one of the pairs 3,$4 ; 3,7 ; 4,6 ; 6,7$.

5. Cases when $n$ and $m$ are Even. When $n$ and $m$ are even, the problem does not reduce to the case $n=1$ as in $\$ 3$. Consider a 3 -set

$$
0, a, b, 2+m x, \quad(x=0,1, \cdots) .
$$

From the sums by three involving $m$ we subtract 2 and get

$$
0,2,4, a, b, a+2, b+2,2 a, a+b, 2 b \text {. }
$$

These ten numbers must include a complete set of residues modulo $m$, whence $m \leqq 10$. Let $m$ be 8 or 10 . Evidently $a$ and $b$ 
are not both even. If $a$ is even and $b$ odd, then $b, b+2, a+b$ are the only odd numbers in (8). Hence $a$ and $b$ are both odd. Then the only odd numbers (8) are

$$
a, b, a+2, b+2 \text {. }
$$

Hence $m \neq 10$. Thus $m=8$. Then (9) are congruent to $1,3,5,7$ in some order. By their sums, $a+b \equiv 2(\bmod 4)$, whence $a \equiv b$. Hence $b \equiv a+4(\bmod 8)$.

Theorem 5. If $m$ is even and $>8$, there is no 3-set (7). If $m=8$, (7) is a 3-set if and only if $a, b$ is one of the pairs 1, 5 or 3, 7. The same result holds if we replace $2+m x$ by $6+m x$ in (7).

The University of Chicago

\section{HYPERTRANSCENDENTAL EXTENSIONS OF PARTIAL DIFFERENTIAL FIELDS*}

BY H. W. RAUDENBUSH, JR.

1. Introduction. In the abstract or formal theory of ordinary algebraic differential equations, $\dagger$ the concept of differential field as defined by Baer $\ddagger$ has a role analogous to that of field in abstract algebra. $\S$ A differential field is a commutative field closed with respect to a formally defined operation called differentiation. The defining rules for differentiation are taken from the elementary properties of derivatives of functions of a single variable. In this paper, with an abstract theory of partial differential equations in mind, we define partial differential fields, selecting the defining rules for differentiation from the elementary properties of partial derivatives of functions of several variables.

* Presented to the Society, March 31, 1934.

$\dagger$ Raudenbush, Differential fields and ideals of differential forms, Annals of Mathematics, vol. 34 (1933), pp. 509-517; and Ideal theory and algebraic differential equations, Transactions of this Society, vol. 36 (1934), pp. 361-368. Also, O. Ore, Formale Theorie der linearen Differentialgleichungen, Journal für Mathematik, vol. 167 (1933), pp. 221-234, and vol. 168 (1934), pp. 233 252.

$\ddagger$ R. Baer, Algebraische Theorie der differentierbaren Funktionenkörper I, Heidelberger Sitzungsberichte, 1927-28.

$\S$ For the terms and theorems of abstract algebra, see van der Waerden, Moderne Algebra. 